\title{
A New Class of Roche Lobe-filling Hot Subdwarf Binaries
}

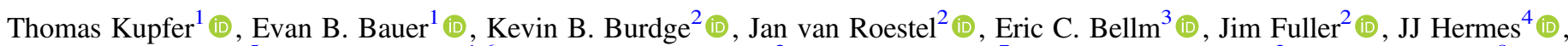 \\ Thomas R. Marsh ${ }^{5}$ (1) , Lars Bildsten ${ }^{1,6}$, Shrinivas R. Kulkarni ${ }^{2}$ (1) E. S. Phinney ${ }^{7}$ (1), Thomas A. Prince ${ }^{2}$ (1) Paula Szkody $^{8}$ (1),

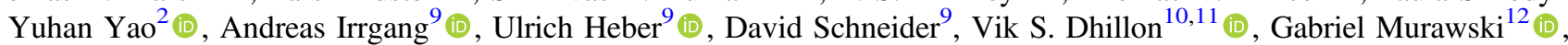

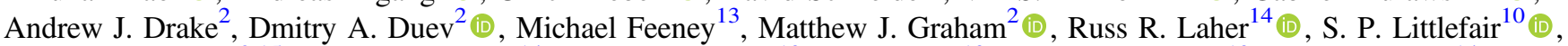 \\ A. A. Mahabal ${ }^{2,15}$ (1), Frank J. Masci ${ }^{14}$ (1) Michael Porter $^{13}$, Dan Reiley ${ }^{13}$, Hector Rodriguez ${ }^{13}$, Ben Rusholme ${ }^{14}$ (1), \\ David L. Shupe ${ }^{14}$ (D), and Maayane T. Soumagnac ${ }^{16,17}$ (iD) \\ ${ }^{1}$ Kavli Institute for Theoretical Physics, University of California, Santa Barbara, CA 93106, USA; tkupfer@ucsb.edu \\ ${ }^{2}$ Division of Physics, Mathematics and Astronomy, California Institute of Technology, Pasadena, CA 91125, USA \\ ${ }^{3}$ DIRAC Institute, Department of Astronomy, University of Washington, 3910 15th Avenue NE, Seattle, WA 98195, USA \\ ${ }^{4}$ Department of Astronomy, Boston University, 725 Commonwealth Avenue, Boston, MA 02215, USA \\ ${ }^{5}$ Department of Physics, University of Warwick, Coventry CV4 7AL, UK \\ ${ }^{6}$ Department of Physics, University of California, Santa Barbara, CA 93106, USA \\ ${ }^{7}$ Theoretical Astrophysics, 350-17, California Institute of Technology, Pasadena CA 91125, USA \\ ${ }^{8}$ University of Washington, Department of Astronomy, Box 351580, Seattle, WA 98195, USA \\ ${ }^{9}$ Dr. Karl Remeis-Observatory \& ECAP, Astronomical Institute, Friedrich-Alexander University Erlangen-Nuremberg (FAU), Sternwartstr. 7, D-96049 Bamberg, \\ Germany \\ ${ }^{10}$ Department of Physics \& Astronomy, University of Sheffield, Sheffield S3 7RH, UK \\ ${ }^{11}$ Instituto de Astrofísica de Canarias, Via Lactea s/n, La Laguna, E-38205 Tenerife, Spain \\ ${ }^{12}$ Gabriel Murawski Private Observatory (SOTES), Poland \\ ${ }^{13}$ Caltech Optical Observatories, California Institute of Technology, Pasadena, CA 91125, USA \\ ${ }^{14}$ IPAC, California Institute of Technology, 1200 E. California Boulevard, Pasadena, CA 91125, USA \\ ${ }^{15}$ Center for Data Driven Discovery, California Institute of Technology, Pasadena, CA 91125, USA \\ ${ }^{16}$ Lawrence Berkeley National Laboratory, 1 Cyclotron Road, Berkeley, CA 94720, USA \\ ${ }^{17}$ Department of Particle Physics and Astrophysics, Weizmann Institute of Science, Rehovot 76100, Israel \\ Received 2020 June 9; revised 2020 July 6; accepted 2020 July 8; published 2020 July 23
}

\begin{abstract}
We present the discovery of the second binary with a Roche lobe-filling hot subdwarf transferring mass to a white dwarf (WD) companion. This 56 minute binary was discovered using data from the Zwicky Transient Facility. Spectroscopic observations reveal an He-sdOB star with an effective temperature of $T_{\text {eff }}=33,700 \pm 1000 \mathrm{~K}$ and a surface gravity of log $(g)=5.54 \pm 0.11$. The GTC+HiPERCAM light curve is dominated by the ellipsoidal deformation of the He-sdOB star and shows an eclipse of the He-sdOB by an accretion disk as well as a weak eclipse of the WD. We infer a He-sdOB mass of $M_{\mathrm{sdOB}}=0.41 \pm 0.04 M_{\odot}$ and a WD mass of $M_{\mathrm{WD}}=0.68 \pm 0.05 M_{\odot}$. The weak eclipses imply a WD blackbody temperature of $63,000 \pm 10,000 \mathrm{~K}$ and a radius $R_{\mathrm{WD}}=0.0148 \pm 0.0020 R_{\odot}$ as expected for a WD of such high temperature. The He-sdOB star is likely undergoing hydrogen shell burning and will continue transferring mass for $\approx 1 \mathrm{Myr}$ at a rate of $10^{-9} M_{\odot} \mathrm{yr}^{-1}$, which is consistent with the high WD temperature. The hot subdwarf will then turn into a WD and the system will merge in $\approx 30$ Myr. We suggest that Galactic reddening could bias discoveries toward preferentially finding Roche lobe-filling systems during the short-lived shell-burning phase. Studies using reddeningcorrected samples should reveal a large population of helium core-burning hot subdwarfs with $T_{\text {eff }} \approx 25,000 \mathrm{~K}$ in binaries of 60-90 minutes with WDs. Though not yet in contact, these binaries would eventually come into contact through gravitational-wave emission and explode as a subluminous thermonuclear supernova or evolve into a massive single WD.
\end{abstract}

Unified Astronomy Thesaurus concepts: B subdwarf stars (129); Stellar evolution (1599); White dwarf stars (1799); Compact binary stars (283); Stellar accretion (1578)

\section{Introduction}

Subdwarf B stars (sdBs) are stars of spectral type B with luminosities below the main sequence. The formation mechanism and evolution of sdBs are still debated, although most sdBs are likely helium (He)-burning stars with masses $\approx 0.5 M_{\odot}$ and thin hydrogen envelopes (Heber 1986, 2009, 2016). A large fraction are found in binary systems (Maxted et al. 2001; Napiwotzki et al. 2004 ) and the most compact ones have periods $\lesssim 1 \mathrm{hr}$ (e.g., Vennes et al. 2012; Geier et al. 2013; Kupfer et al. 2017b, 2017a, 2020). Systems with orbital periods $\lesssim 2 \mathrm{~h}$ at the exit of the last common envelope will overflow their Roche lobe while the hot subdwarf is still burning helium (e.g., Savonije et al. 1986; Tutukov \& Fedorova 1989; Tutukov \& Yungelson 1990; Iben \& Tutukov 1991; Yungelson 2008; Piersanti et al. 2014; Brooks et al. 2015).
So far only a few hot subdwarf binaries have been identified that will start mass transfer while the hot subdwarf is still burning helium in its core. The first discovered system is CD-30 11223 , which has an orbital period of $P_{\text {orb }}=70.5$ minutes and will start mass transfer in $\approx 40 \mathrm{Myr}$ (Vennes et al. 2012; Geier et al. 2013).

Most recently Kupfer et al. (2020) discovered ZTF J2130 +4420 , the first system where the sdOB fills its Roche lobe and has started mass transfer to its white dwarf (WD) companion. In this Letter, we report the discovery of ZTF J205515.98+465106.5 (hereafter ZTF J2055+4651), the second confirmed member of Roche lobe-filling hot subdwarf stars in compact binaries. ZTF J2055+4651 was discovered in a period search using the conditional entropy period-finding algorithm (Graham et al. 2013) of all Pan-STARRS objects with colors of $g-r<0.2$ and $r-i<0.2$, and more than $50 \mathrm{ZTF}$ measurements at the time, as 
described in Burdge et al. (2019) and Coughlin et al. (2020). Conditional entropy is an information-theory-based algorithm that seeks to find the period that produces the most ordered (lowest entropy) arrangement of data points in the phase-folded light curve while also accounting for the phase space coverage of the data. Independently, on 2019 July 19, Gabriel Murawski reported the object to the International Variable Star Index (VSX). ${ }^{18}$ Also, Sandoval et al. (2020) report the absence of X-ray emission from $3.7 \mathrm{ks}$ Swift observations and found a limit for the $\mathrm{X}$-ray flux of $<1 \times 10^{-13} \mathrm{erg} \mathrm{s}^{-1} \mathrm{~cm}^{-2}$ leading to a limit of $<6 \times 10^{31} \mathrm{erg} \mathrm{s}^{-1}$ at a distance of $2170 \mathrm{pc}$ (Bailer-Jones et al. 2018). We present the system properties, compare them to ZTF J2130+4420, and discuss possible implications for this new class of Roche lobe-filling hot subdwarf binaries.

\section{Observations}

As part of the Zwicky Transient Facility (ZTF), the Palomar 48-inch (P48) telescope images the sky every clear night. ZTF J2055+4651 was discovered as part of a dedicated highcadence ZTF survey at low Galactic latitudes (Bellm et al. 2019a; Graham et al. 2019), which either observed one field or alternated between two adjacent fields continuously for $\approx 1.5-3$ hours on two to three consecutive nights in the ZTF$r$ band (Bellm et al. 2019b). The high-cadence data were complemented by data from the ZTF public survey that was made available after data release 2 on 2019 December 11 . Image processing of ZTF data is described in full detail in Masci et al. (2019). The ZTF light curve of ZTF J2055+4651 consists of 224 observations in ZTF- $g$ and 496 observations in ZTF- $r$.

Additionally on 2019 September 5, ZTF J2055+4651 was observed with the Gran Telescopio Canarias (GTC) using HiPERCAM, which is a five-beam imager equipped with frame-transfer CCDs allowing the simultaneous acquisition of $u_{s}, g_{s}, r_{s}, i_{s}$, and $z_{s}{ }^{19}$ band images at a rate of up to 1000 frames per second (Dhillon et al. 2016, 2018). ZTF J2055+4651 was observed at a $3 \mathrm{~s}$ cadence with a dead time of $10 \mathrm{~ms}$ for 1130 frames with HiPERCAM. The run lasted $1 \mathrm{hr}$, covering a little more than one 56 minute binary orbit. The cadence in $u_{s}$ and $z_{s}$ alone was a factor of 3 slower than the other bands to optimize signal to noise. The data were reduced using the dedicated HiPERCAM pipeline, ${ }^{20}$ including debiasing and flat-fielding. Differential photometry was also performed. Due to technical problems the $i_{s}$-band light curve could not be used for the analysis.

On 2019 July 30 a sequence of five optical spectra was obtained with the Shane telescope and the KAST spectrograph at Lick observatory using a low-resolution mode $(R \approx 1200)$. The spectra were taken consecutively and had an exposure time of 12 minutes. Data reduction was performed with the standard IRAF packages. Although 12 minutes covers $1 / 5$ of the orbit we detected large radial velocity shifts between the spectra, confirming the binary nature.

Additionally, on 2019 September 24 and 2019 September 25 phase-resolved spectroscopy of ZTF J2055+4651 was obtained using the Gemini-North Telescope, using Gemini Multi-Object Spectrograph (GMOS) in long-slit mode with the B600 grating

\footnotetext{
18 https://www.aavso.org/vsx/index.php?view = detail.top\&oid $=838260$

19 HiPERCAM uses high-throughput versions of the SDSS filters known as Super-SDSS filters, and we denote these filters $u_{s}, g_{s}, r_{s}$, etc.

${ }^{20}$ https://github.com/HiPERCAM/
}

$(R \approx 1400)$. We obtained a total of 64 spectra with an exposure time of 3 minutes per spectrum. CuAr arc exposures were taken at the position of the target before and after each observing sequence to account for telescope flexure. The data were reduced using the IRAF package for GMOS.

\section{Period Finding and Spectroscopic Analysis}

As we followed the same procedure as described in Kupfer et al. (2020), we only briefly describe the major steps here. To derive the orbital period of the system, we use the ZTF light curve with its multi-month baseline in combination with the HiPERCAM light curve. The Gatspy (Vanderplas 2015; VanderPlas \& Ivezić 2015) module that utilizes the LombScargle periodogram (Lomb 1976; Scargle 1982) was used for period finding, yielding $P_{\text {orb }}=56.34785(26)$ minutes. Using the parameter from the best light-curve fit (see Section 4) and only fitting the zero-point of the ephemeris $\left(T_{o}\right)$ to the HiPERCAM light curves, we find an ephemeris of

$$
T_{o}(\mathrm{BMJD})=58731.9639(90)+0.03913045(18) E,
$$

where $E$ corresponds to the epoch.

We find spectral features of hydrogen and neutral (He I) and ionized (He II) helium lines typical for sdOB stars. By fitting the rest-wavelength corrected average Gemini spectrum with metal-free NLTE model spectra (Stroeer et al. 2007), we determine $T_{\text {eff }}=33,700 \pm 1000 \mathrm{~K}, \log (g)=5.54 \pm 0.11$, $\log y=\log [n(\mathrm{He}) / n(\mathrm{H})]=0.06 \pm 0.05$, and $v_{\text {rot }} \sin i=201 \pm$ $30 \mathrm{~km} \mathrm{~s}^{-1}$. Additionally, we measured atmospheric parameters using NLTE models computed with TLUSTY (Hubeny \& Lanz 1995) that include typical hot subdwarf abundances of nitrogen, carbon, and oxygen (Naslim et al. 2013). We find fully consistent results with our metal-free NLTE models. Figure 1 shows the fit to the Gemini spectrum. The occurrence of both He I and He II as well as the increased helium abundance $\log y>0$ classifies the hot subdwarf as an He-sdOB star (see Heber 2016).

We folded the individual Gemini spectra on the ephemeris shown in Equation (1) into 20 phase-bins and coadded individual spectra observed at the same binary phase. This leads to a signal to noise per phase-bin of $\approx 30$. We used the FITSB2 routine (Napiwotzki et al. 2004; Geier et al. 2011) to then measure radial velocities. Assuming circular orbits, a sine curve was fitted to the folded radial velocity (RV) data points (Figure 2) excluding the data points around Phase 0.8-0.2. We find a velocity semi-amplitude $K=404.0 \pm 11.0 \mathrm{~km} \mathrm{~s}^{-1}$. Similar to ZTF J2130, the velocity deviates significantly from a pure sine curve around the phase when the sdOB is farthest away from the observer, which can be explained with the Rossiter-McLaughlin effect. The red curve in Figure 2 show the residuals predicted from the Rossiter-McLaughlin effect calculated from our best-fitting light-curve model.

\section{Light-curve Analysis and System Parameter}

The LCURVE code was used to perform the light-curve analysis of the HiPERCAM $u_{s}, g_{s}, r_{s}$, and $z_{s}$ light curves (Copperwheat et al. 2010). We assume a Roche lobe-filling sdOB star, a hot $\mathrm{WD}$, and an irradiated disk around the WD. The passband specific beaming parameter $B\left(F_{\lambda}=F_{0, \lambda}\left[1-B \frac{v_{r}}{c}\right]\right.$; see Bloemen et al. 2011) was calculated following the approximation from Loeb \& Gaudi (2003). We use $B=1.7,1.5,1.37$, and 1.25 for $u_{s}, g_{s}, r_{s}$, and $z_{s}$, respectively. The passband specific gravity-darkening $(\beta)$ and limb-darkening $\left(a_{1}, a_{2}, a_{3}, a_{4}\right)$ were fixed to the theoretical 

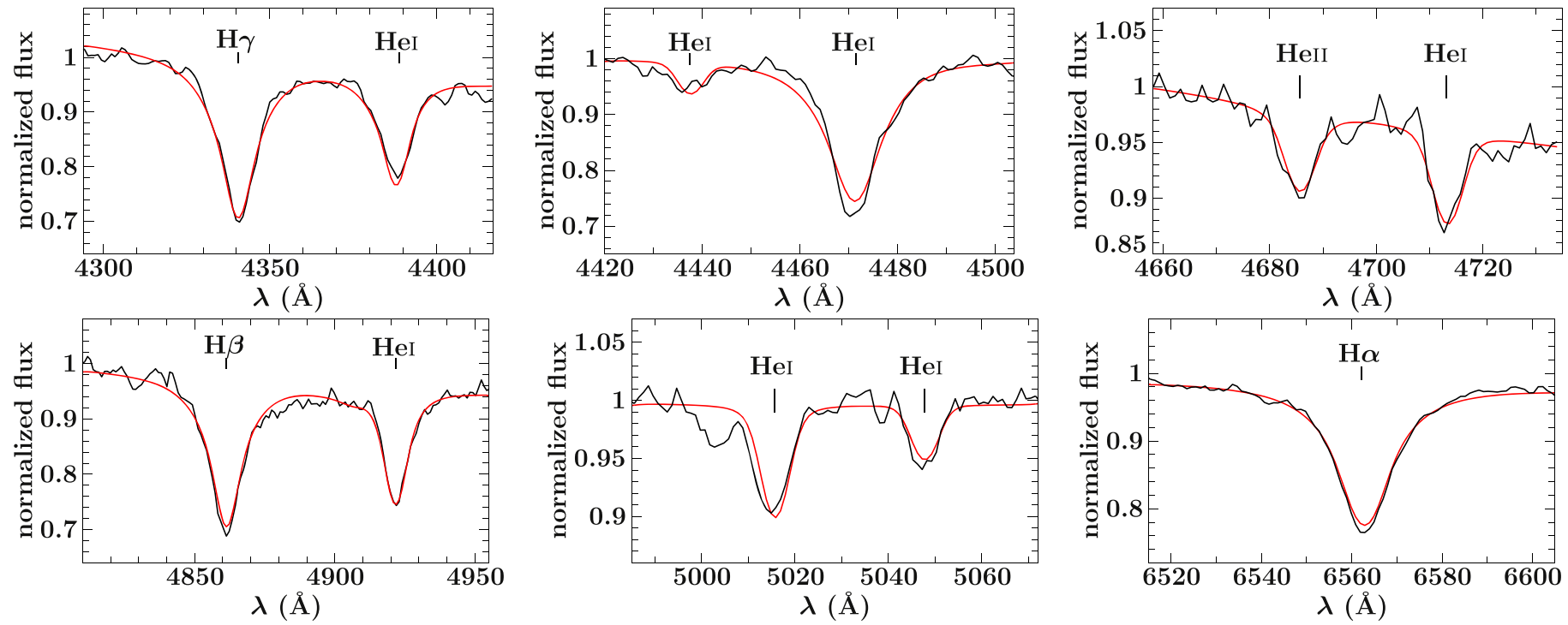

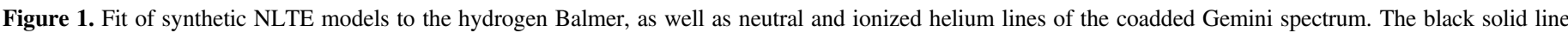
corresponds to the spectrum and red solid line to the fit.

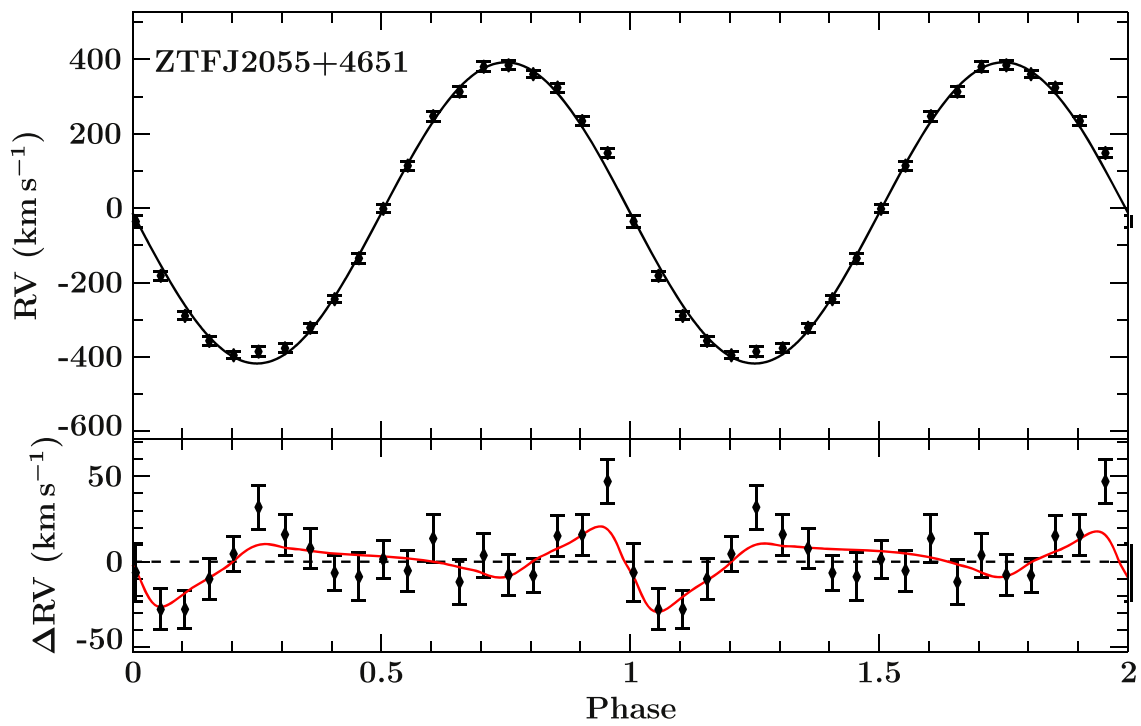

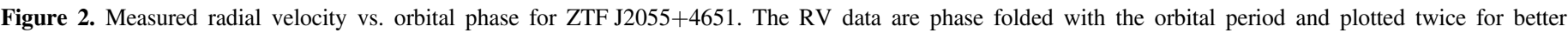

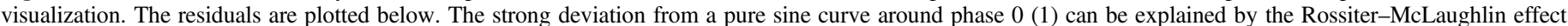

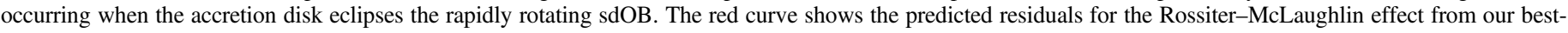
fitting model (see Section 4).

values from Claret \& Bloemen (2011) for $T_{\text {eff }}=35,000 \mathrm{~K}$ and $\log (g)=5.00$ and solar metallicity. We used $\beta=0.54, a_{1}=$ 0.93, $a_{2}=-0.98, a_{3}=0.85$, and $a_{4}=-0.29$ for $u_{s} ; \beta=0.42$, $a_{1}=0.96, a_{2}=-1.05, a_{3}=0.87$, and $a_{4}=-0.28$ for $g_{s}$; $\beta=0.41, a_{1}=0.90, a_{2}=-1.11, a_{3}=0.94$, and $a_{4}=-0.31$ for $r_{s}$; and $\beta=0.39, a_{1}=0.83, a_{2}=-1.20, a_{3}=1.09$, and $a_{4}=-0.38$ for $z_{s}$. We also tried to set the limb- and gravitydarkening parameters according to the tables of Claret et al. (2020) for hydrogen-rich atmospheres and found no significant difference in the final results. In addition, to account for any residual airmass effect we added a first-order polynomial. The best value of $\chi^{2}$ for this model was around 3500 for 3012 points, which also includes a weak eclipse of the hot WD. Although the eclipse is weak $(\leqslant 1 \%)$, the $\chi^{2}$ for the non-eclipsing solution is 3900 , which is statistically significantly worse compared to the solution with the weak eclipse. For the final model we normalized the errors in the data to account for the small additional residuals and obtain a reduced $\chi^{2} \approx 1$. To determine the uncertainties in the parameters we combine LCURVE with emcee (Foreman-Mackey et al. 2013), an implementation of a Markov Chain Monte Carlo sampler that uses a number of parallel chains to explore the solution space. We use 256 chains and let them run until the chains stabilized to a solution, which took approximately 1500 generations. Figure 3 shows the best fit for each HiPERCAM band.

Although ZTF J2055+4651 is a single-lined binary, we can still constrain the masses and radii of the two stars by combining the light-curve modeling with the spectroscopic fitting. Parameters derived in this way by a simultaneous fit to the HiPERCAM $u_{s}$, $g_{s}, r_{s}$, and $z_{s}$ light curves are summarized in Table 1 .

Our solution converges on a mass ratio of $q=M_{1} / M_{2}=$ $0.60 \pm 0.03$, with individual masses of $M_{\mathrm{He}-\mathrm{sdOB}}=0.41 \pm$ $0.04 \mathrm{M}_{\odot}$ and $M_{\mathrm{WD}}=0.68 \pm 0.05 \mathrm{M}_{\odot}$. The inclination is $i=$ $83.4 \pm 1.0$ and the He-sdOB is Roche lobe-filling and has a 

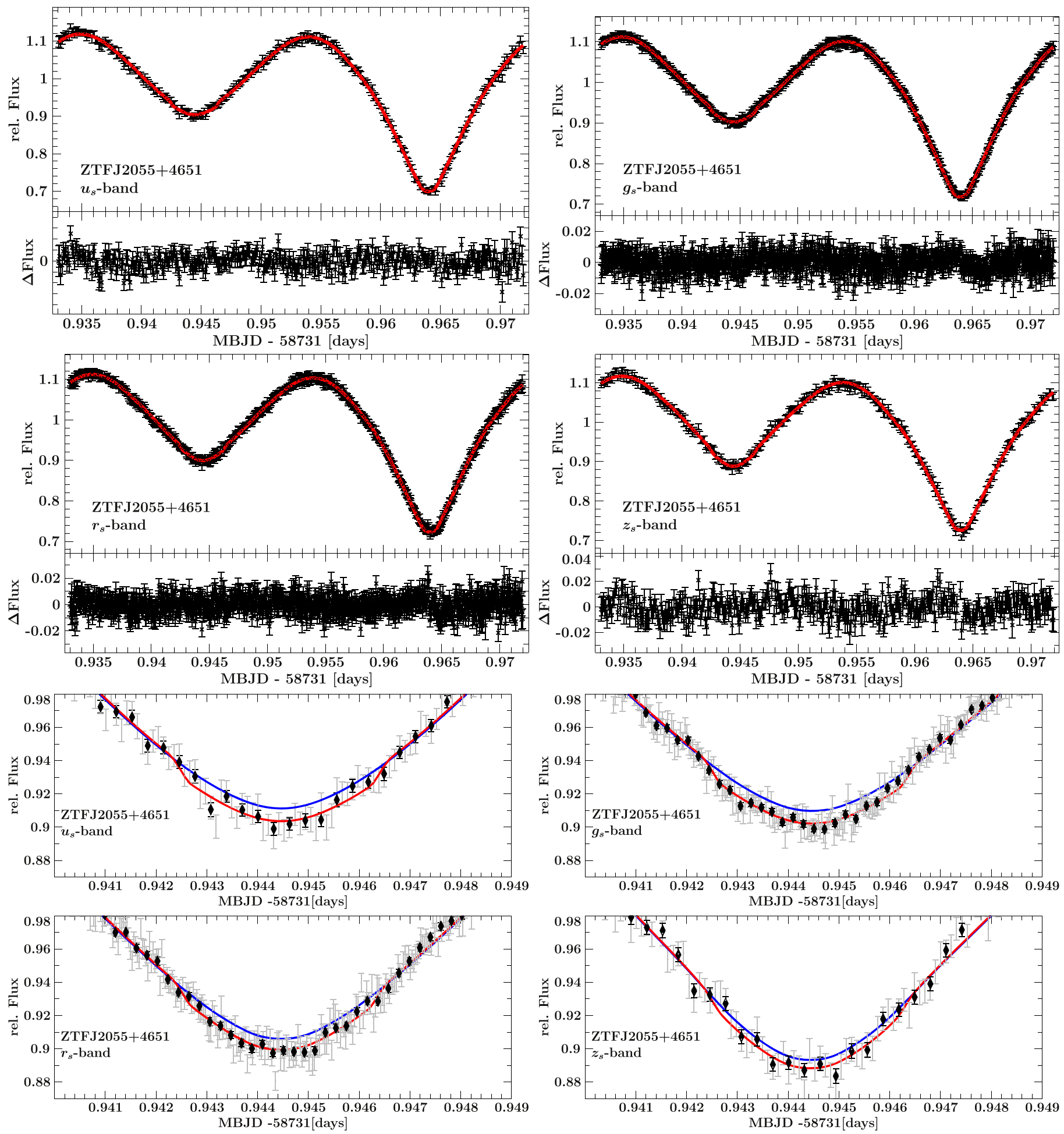

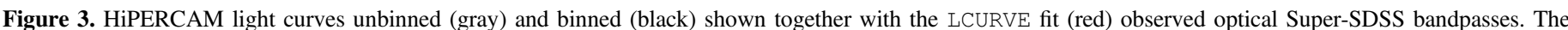

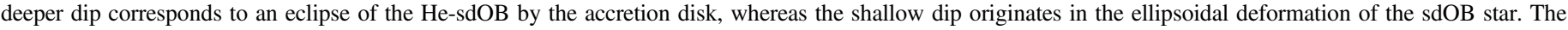

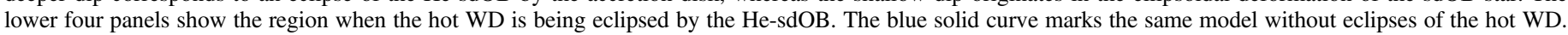

volumetric-corrected radius of $R_{\text {sdOB }}=0.17 \pm 0.01 R_{\odot}$ (Table 1$)$. We find that the He-sdOB would have a projected rotational velocity $v_{\text {rot }} \sin i=215 \pm 6 \mathrm{~km} \mathrm{~s}^{-1}$ if synchronized to the orbit, consistent with the observed $v_{\text {rot }} \sin i=201 \pm 30 \mathrm{~km} \mathrm{~s}^{-1}$. We detect a weak eclipse of the hot WD that constrains its properties (Figure 3). We derive a blackbody temperature of $63,000 \pm$ $10,000 \mathrm{~K}$ for the WD, with a radius of $0.0148 \pm 0.0020 R_{\odot}$, which is $10 \%-50 \%$ larger than a fully degenerate WD. This is consistent with predictions for hot WDs as shown in Romero et al. (2019), who predict $25 \%$ increased radius for a carbon-oxygen WD with $T_{\text {eff }}=60,000 \mathrm{~K}$ and a hydrogen layer of $M=10^{-4} M_{\odot}$.

The spectral energy distribution (SED) in combination with the Gaia parallax allows for an independent estimate of the stellar parameters of the hot subdwarf. To this end, we first 
Table 1

Measured and Derived Parameters for ZTF J2055+4651

\begin{tabular}{|c|c|c|}
\hline & & \\
\hline$\overline{\text { Right }}$ ascension $^{\text {a }}$ & 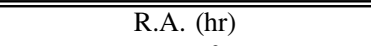 & $20: 55: 15.98$ \\
\hline Declination $^{\mathrm{a}}$ & decl. $\left({ }^{\circ}\right)$ & $+46: 51: 06.46$ \\
\hline Magnitude $^{\mathrm{b}}$ & $g$ (mag) & $17.72 \pm 0.04$ \\
\hline Parallax $^{\mathrm{a}}$ & $\varpi$ (mas) & $0.432 \pm 0.098$ \\
\hline Distance & $d(\mathrm{kpc})$ & $2.3_{-0.4}^{+0.7}$ \\
\hline reddening $^{c}$ & $E(g-r)(\mathrm{mag})$ & $0.46 \pm 0.03$ \\
\hline $\begin{array}{l}\text { Absolute } \\
\text { magnitude }\end{array}$ & $M_{\mathrm{g}}(\mathrm{mag})$ & $4.4 \pm 0.6$ \\
\hline $\begin{array}{l}\text { (reddening } \\
\quad \text { corrected }^{\mathrm{d}}\end{array}$ & & \\
\hline $\begin{array}{l}\text { Proper motion } \\
\text { (R.A.) }^{\mathrm{a}}\end{array}$ & $\mu_{\alpha} \cos (\delta)\left(\right.$ mas yr $\left.^{-1}\right)$ & $-3.33 \pm 0.17$ \\
\hline $\begin{array}{l}\text { Proper motion } \\
\quad(\text { decl. })^{\mathrm{a}}\end{array}$ & $\mu_{\delta}\left(\operatorname{mas~yr}^{-1}\right)$ & $-5.160 \pm 0.167$ \\
\hline Atmospheric and $\mathrm{O}$ & ital Parameters of the sdOB from & pectroscopic Fits \\
\hline $\begin{array}{l}\text { Effective } \\
\text { temperature }\end{array}$ & $T_{\text {eff }}(\mathrm{K})$ & $33,700 \pm 1000$ \\
\hline Surface gravity & $\log (g)$ & $5.54 \pm 0.11$ \\
\hline Helium abundance & $\log y$ & $0.06 \pm 0.05$ \\
\hline $\begin{array}{l}\text { Projected rotational } \\
\text { velocity }\end{array}$ & $v_{\mathrm{rot}} \sin i\left(\mathrm{~km} \mathrm{~s}^{-1}\right)$ & $201 \pm 30$ \\
\hline $\begin{array}{l}\text { RV semi-ampl- } \\
\text { itude (sdOB) }\end{array}$ & $K\left(\mathrm{~km} \mathrm{~s}^{-1}\right)$ & $404.0 \pm 11.0$ \\
\hline System velocity & $\gamma\left(\mathrm{km} \mathrm{s}^{-1}\right)$ & $-20.0 \pm 6.0$ \\
\hline $\begin{array}{l}\text { Binary mass } \\
\text { function }\end{array}$ & $f_{\mathrm{m}}\left(M_{\odot}\right)$ & $0.267 \pm 0.022$ \\
\hline Derived Parameter & om Light-curve Fit Combined with & Ppectroscopic Results \\
\hline $\begin{array}{l}\text { Ephemeris zero- } \\
\text { point }\end{array}$ & $T_{0}(\mathrm{MBJD})$ & $58731.9639(90)$ \\
\hline Orbital period & $P_{\text {orb }}(\min )$ & $56.34785(26)$ \\
\hline Mass ratio & $q=\frac{M_{\mathrm{sdOB}}}{M_{\mathrm{WD}}}$ & $0.60 \pm 0.03$ \\
\hline sdOB mass & $M_{\mathrm{sdOB}}\left(M_{\odot}\right)$ & $0.41 \pm 0.04$ \\
\hline sdOB radius & $R_{\mathrm{sdOB}}\left(R_{\odot}\right)$ & $0.17 \pm 0.01$ \\
\hline WD mass & $M_{\mathrm{WD}}\left(M_{\odot}\right)$ & $0.68 \pm 0.05$ \\
\hline WD radius & $R_{\mathrm{WD}}\left(M_{\odot}\right)$ & $0.0148 \pm 0.0020$ \\
\hline Orbital inclination & $i\left(^{\circ}\right)$ & $83.4 \pm 1.0$ \\
\hline Separation & $a\left(R_{\odot}\right)$ & $0.50 \pm 0.01$ \\
\hline Derived Parameters & Spectrophotometry and Para & \\
\hline Color excess & $E(44-55)$ & $0.559 \pm 0.021 \mathrm{mag}$ \\
\hline Angular diameter & $\log (\Theta(\mathrm{rad}))$ & $-11.479 \pm 0.011$ \\
\hline sdOB radius & $R=\Theta /(2 \varpi)\left(R_{\odot}\right)$ & $0.17_{-0.04}^{+0.05}$ \\
\hline sdOB mass & $M=g R^{2} / G\left(M_{\odot}\right)$ & $0.37_{-0.14}^{+0.29}$ \\
\hline Luminosity & $L / L_{\odot}=\left(R / R_{\odot}\right)^{2}\left(T_{\mathrm{eff}} / \mathrm{T}_{\mathrm{eff}, \odot}\right)^{4}$ & $34_{-12}^{+24}$ \\
\hline
\end{tabular}

Notes.

a Taken from Gaia DR2, epoch 2015.5 (Gaia Collaboration et al. 2016, 2018).

b Taken from PanSTARRS DR1 (Chambers et al. 2016).

${ }^{c}$ Taken from 3D extinction maps (Green et al. 2019).

${ }^{\mathrm{d}}$ Reddening corrected with 3D extinction maps from Green et al. (2019).

e The given uncertainties are single-parameter $1 \sigma$ confidence intervals based on $\chi^{2}$ statistics. A generic excess noise of 0.026 mag has been added in quadrature to all photometric measurements to achieve a reduced $\chi^{2}$ of unity at the best fit.

construct the observed SED from Gaia (Evans et al. 2018) and PanSTARRS photometry (Chambers et al. 2016). ${ }^{21}$ Using the atmospheric parameters derived by spectroscopy, a synthetic SED is computed with an updated version of ATLAS12 (Kurucz 1996; Irrgang et al. 2018), which is then matched to the observed SED by fitting the angular diameter $\Theta$ as a

\footnotetext{
${ }^{21}$ Based on averages of 223 epochs of good Gaia observations and of 80 epochs of PanSTARRS observations.
}

distance scaling factor and $E(44-55)$ as an interstellar reddening parameter (see Heber et al. 2018 for details). The interstellar extinction law by Fitzpatrick et al. (2019) is used. The results are summarized in Table 1 and visualized in Figure 4. As expected for a star in the Galactic disk, reddening is large $(E(44-55)=0.559 \pm 0.021 \mathrm{mag})$.

In combination with the Gaia parallax $\varpi$ and the atmospheric parameters $\left(T_{\text {eff }}\right.$ and $\left.\log (g)\right)$ the angular diameter allows us to determine the stellar parameters $\left(R_{\mathrm{sdOB}}, M_{\mathrm{sdOB}}\right.$, and luminosity $\left.L_{\mathrm{sdOB}}\right)$. Results are listed Table 1 . The mass derived for the hot subdwarf agrees well with that derived from the light curve, although the uncertainty of the former is large because of the $\approx 25 \%$ parallax error and $0.11 \mathrm{dex} \log (g)$ error.

Using 3D extinction maps provided by Green et al. $(2019)^{22}$ toward the direction of ZTF J2055+4651, we find a reddening of $E(g-r)=0.46 \pm 0.03$ at a distance of $2.3_{-0.4}^{+0.7} \mathrm{kpc}$, which is somewhat lower than the reddening derived by the SED analysis. Nonetheless, ZTF J2055+4651 is highly reddened and has a PanSTARRS color of $g-r=0.07$ mag (Chambers et al. 2016). Such a color puts it in the regime of A- to F-type stars with $T_{\mathrm{eff}} \approx 10,000 \mathrm{~K}$, which is significantly cooler than typical hot subdwarfs. With the 3D map extinction maps corrected magnitude, we find an absolute magnitude of $M_{\mathrm{g}}=4.4 \pm 0.6 \mathrm{mag}$, consistent with a hot subdwarf star (Geier et al. 2019).

To put constraints on the population origin of ZTF J2055+ 4651 we calculated its kinematics. The proper motions of the system is taken from the Gaia data release 2 catalog (Gaia Collaboration et al. 2018; $\mu_{\alpha} \cos (\delta)=-3.326 \pm 0.167 \mathrm{mas} \mathrm{yr}^{-1}$, $\left.\mu_{\delta}=-5.160 \pm 0.167 \mathrm{mas} \mathrm{yr}^{-1}\right)$. We followed the same approach as described in Kupfer et al. (2020) following Odenkirchen \& Brosche (1992) and Pauli et al. (2006). We use the Galactic potential of Allen \& Santillan (1991) as revised by Irrgang et al. (2013). The orbit was integrated from the present to $3 \mathrm{Gyr}$ into the past. ZTF J2055+4651 moves between a distance of 8.6 to $11.6 \mathrm{kpc}$ from the Galactic center with an eccentricity of 0.07 within a height of $50 \mathrm{pc}$ of the Galactic equator. Exactly as for the other Roche lobe-filling hot subdwarf binary, ZTF J2130+ 4420 , ZTF J2055+4651 is a member of the Galactic thin-disk population.

\section{Discussion \\ 5.1. Evolution}

Following the same prescription as described in full detail in Section 6.2 in Kupfer et al. (2020) we employed MESA version 12115 (Paxton et al. 2011, 2013, 2015, 2018, 2019) to construct evolutionary models for ZTF J2055+4651. To match the observed properties of ZTF J2055+4651, we start with a MESA model of a progenitor star with a mass of $2.9 M_{\odot}$, which has a main-sequence lifetime of $350 \mathrm{Myr}$. Once the mainsequence star starts to evolve onto the red giant branch (RGB) $\mathrm{He}$ core burning starts under nondegenerate conditions. We remove most of the hydrogen-rich envelope, leaving a He coreburning sdB with $T_{\text {eff }} \approx 25,000 \mathrm{~K}$, a core mass of $0.36 M_{\odot}$, and a thin $\mathrm{H} / \mathrm{He}$ envelope of $0.03 M_{\odot}$. The envelope is defined by where the $\mathrm{H}$ mass fraction is above $0.01 M_{\odot}$. This subdwarf model is then placed in a binary with a $0.65 M_{\odot}$ WD with an orbital period of $\approx 140 \mathrm{~min}$. Gravitational-wave radiation removes angular momentum from the binary and shortens the

\footnotetext{
${ }^{22}$ http://argonaut.skymaps.info/
} 


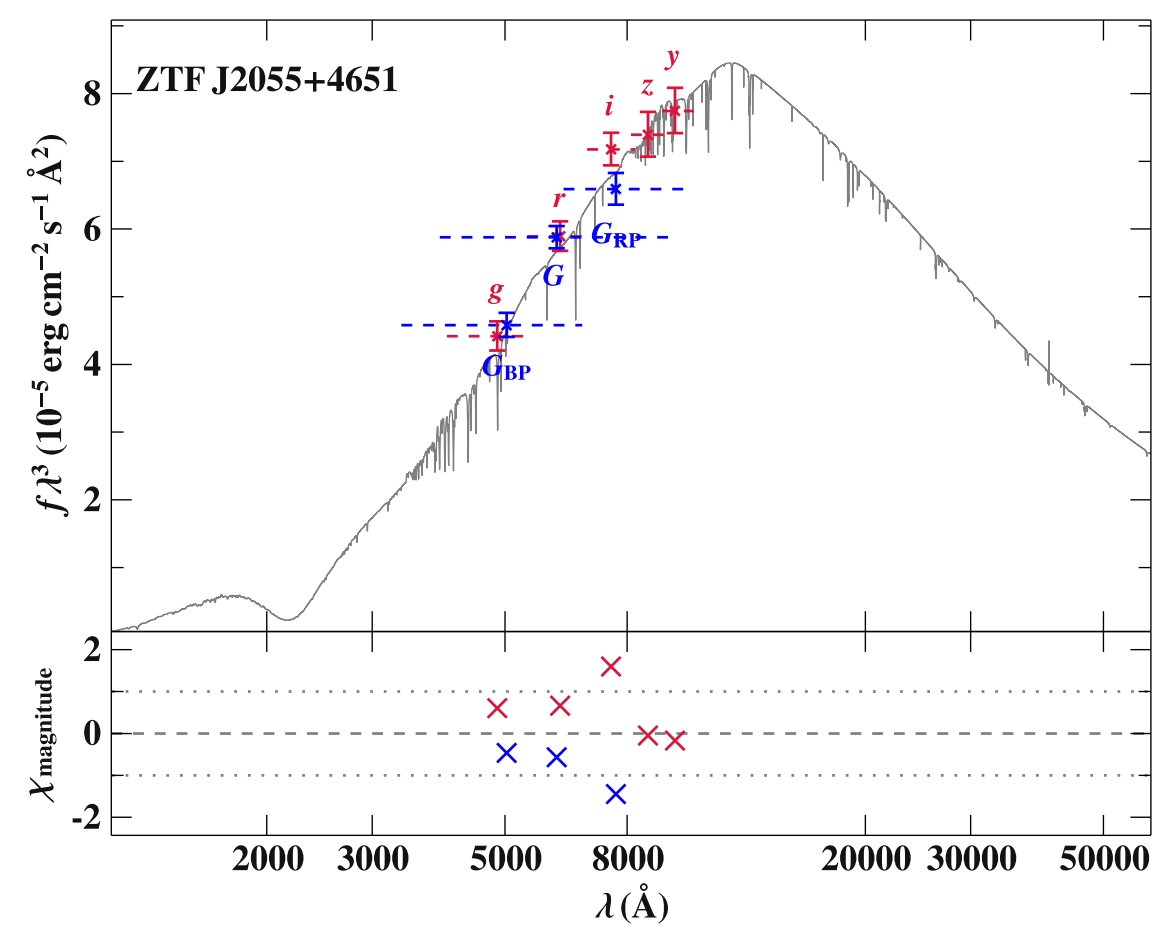

Figure 4. Comparison of synthetic and observed photometry: The top panel shows the SED. The colored data points are filter-averaged fluxes that were converted from observed magnitudes (the respective filter widths are indicated by the dashed horizontal lines), while the gray solid line represents the best-fitting model, i.e., it is based on the parameters from Table 1, degraded to a spectral resolution of $6 \AA$. The flux is multiplied with the wavelength to the power of three to reduce the steep slope of the SED. The bottom panel shows the residual $\chi$, i.e., the difference between synthetic and observed magnitudes divided by the corresponding uncertainties. Only the angular diameter $\Theta$ and the color excess $E(B-V)$ are free fitting parameters. Pan-STARRS1 magnitudes (Chambers et al. (2016) are shown in red and Gaia magnitudes (Evans et al. 2018) with corrections and calibrations from Maíz Apellániz \& Weiler (2018) in blue.

period. The period does not shrink far enough during He core burning, which lasts $\approx 350 \mathrm{Myr}$, that the sdB fills its Roche lobe (see the left panel in Figure 5).

Once He core burning finishes the core contracts and hydrogen burning starts, the radius of the hot subdwarf expands beyond its Roche radius, and mass transfer starts at an orbital period close to the observed orbital period. Mass transfer will continue for $\approx 1 \mathrm{Myr}$ at a rate exceeding $10^{-9} M_{\odot} \mathrm{yr}^{-1}$ until hydrogen shell burning is finished and the star contracts to become a carbonoxygen WD with a thick helium layer and a small residual layer of hydrogen. The high accretion rate will heat the accreting WD significantly (Townsley \& Bildsten 2003). Models predict a $T_{\text {eff }} \approx 50,000 \mathrm{~K}$ for accretion rates of $10^{-9} M_{\odot} \mathrm{yr}^{-1}$ (Burdge et al. 2019) consistent with the high blackbody temperature of the accretor observed in ZTF J2055+4651. Accretion onto the WD companion at this rate will cause unstable hydrogen ignition after $\approx 10^{-4} M_{\odot}$ accumulates, leading to classical novae eruptions (Nomoto 1982; Nomoto et al. 2007; Wolf et al. 2013). This accretion rate predicts a recurrence time of order $10^{5} \mathrm{yr}$ for a total of approximately 10 novae. Our binary model suggests that this system is within the first $\approx 10 \%$ of the $1 \mathrm{Myr}$ accretion phase. At the current state the orbit will shrink with $\dot{P} \approx-6 \times 10^{-13} \mathrm{~s} \mathrm{~s}^{-1}$, which will be detectable after a few years of monitoring. The right panel of Figure 5 shows the evolution of the donor through this mass transfer phase. After accretion has ceased, the orbit of the system will continue to shrink due to the radiation of gravitational waves and the system will merge in $\approx 30$ Myr. Our models predict that there is a substantial He layer of $\approx 0.05 M_{\odot}$ left in the former hot subdwarf and the total mass of the system is relatively high $\left(M_{\text {total }} \approx 1.1 M_{\odot}\right)$. Recent models predict that such a system explodes as a subluminous thermonuclear supernova (Perets et al. 2019; Zenati et al. 2019). If the system avoids a thermonuclear supernova it will merge and could evolve into a rapidly rotating single high-mass carbon-oxygen WD (Saio 2008; Clayton 2012; Schwab 2019).

\subsection{The Accretion Disk}

Our Gemini spectra show no evidence for any disk lines, which limits the accretion disk contribution of the accretion disk to the overall luminosity to $\leqslant 5 \%$. Our models predict an accretion rate of $10^{-9} M_{\odot} \mathrm{yr}^{-1}$. From that we can limit the accretion luminosity to be $\approx 1.5 \mathrm{~L} \odot$ which is significantly smaller than the luminosity of the sdOB star and in agreement with the absence of any signs of the disk in the optical spectra.

Sandoval et al. (2020) reported the nondetection of X-rays in a 3.6 ks observation with the Neil Gehrels Swift Observatory. $\mathrm{X}$-rays from disk-accreting WDs are generally emitted from the boundary layer. We expect that $\approx 50 \%$ of the accretion luminosity is released in the boundary layer. This corresponds to $\approx 3 \times 10^{31} \mathrm{erg} \mathrm{s}^{-1}$, which is $\approx 50 \times$ larger than the $\mathrm{X}$-ray upper limit. This suggests an optically thick boundary layer in ZTF J2055+4651 with most flux emitted in the extreme UV. This has been observed in SS Cyg, where the X-ray luminosity dropped and the extreme UV emission increased during outburst, as the accretion rate increased (Wheatley et al. 2003).

\subsection{Implications for a Population of Roche Lobe-filling Hot Subdwarfs}

Kupfer et al. (2020) presented the first known Roche lobefilling hot subdwarf binary. ZTF J2055+4651 is the second known system and the overall evolution appears to be similar to ZTF J2130+4420. Both systems have $M_{\mathrm{sdOB}}<0.47 M_{\odot}$ hot subdwarf masses and likely evolved from stars with masses 

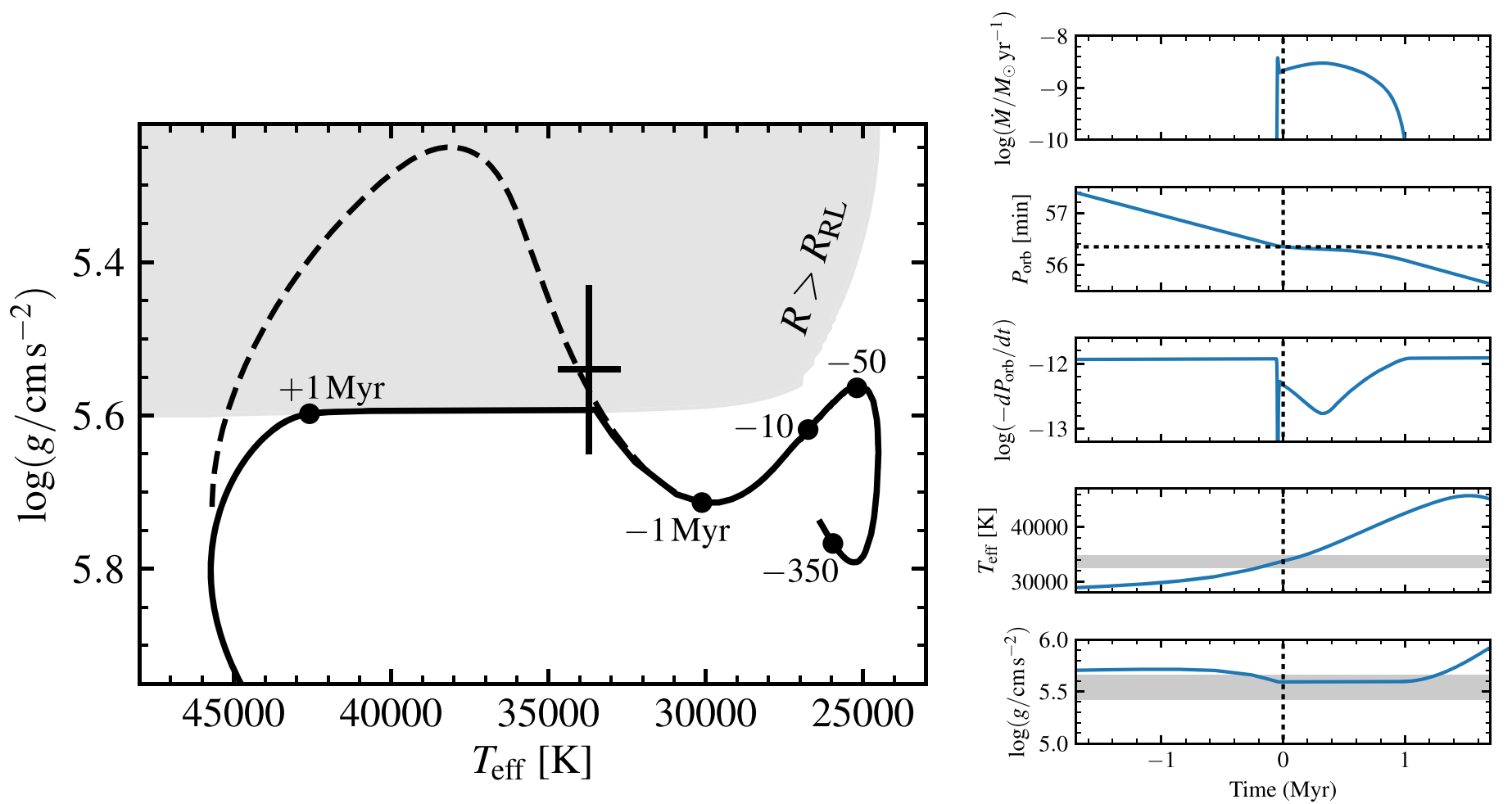

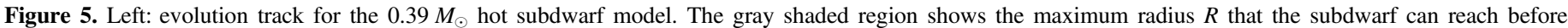

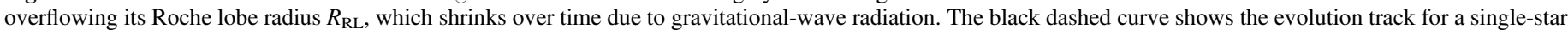

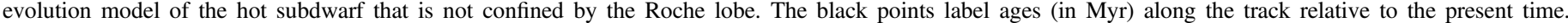

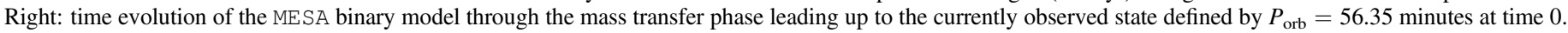
Gray shaded regions in the lower two panels show the measured values of $T_{\text {eff }}$ and $\log (g)$ given in Table 1 .

$>2 M_{\odot}$ and therefore are members of a young stellar population consistent with the observed Galactic kinematics. This result is also consistent with predictions by Han et al. (2003), who found that the most compact hot subdwarf binaries with a compact companion are expected to evolve from mainsequence stars with masses $>2 M_{\odot}$. Their study showed that hot subdwarf binaries formed with orbital periods $\lesssim 2 \mathrm{hr}$ are dominated by systems with progenitor masses $>2 M_{\odot}$.

ZTF J2130+4420 and ZTF J2055+4651 are hotter compared to most known hot subdwarfs and can be best explained as donor stars during the shell-burning phase. Given that the residual hydrogen shell-burning lifetime of the hot subdwarf is a factor of $\approx 10-100$ shorter than its helium core-burning lifetime we expect a population $\approx 10-100$ larger of systems during core burning. We suggest here that Galactic reddening could bias discoveries toward preferentially finding Roche lobe-filling systems during shell burning.

Systems located at low Galactic latitudes will suffer from extinction and reddening. Although the exact extinction is direction dependent, on average the Galactic plane has an extinction of $\approx 1 \mathrm{mag}$ per $\mathrm{kpc}$ in the optical bands where bluer bands are more strongly affected (Gontcharov 2016; Green et al. 2019). This leads to a shift of PanSTARRS $g-r$ colors of $\approx 0.25-0.3$ mag per kpc toward redder color leading to a biased sample of hotter or less distant systems in color-selected samples. Typically hot subdwarfs have PanSTARRS colors of $g-r \approx$ $-0.35 \mathrm{mag}$ (Geier et al. 2019) leading to $g-r \approx 0.15-0.25 \mathrm{mag}$ for a $2 \mathrm{mag}$ extinction at a distance of $2 \mathrm{kpc}$. These are typical colors for F- to G-type main-sequence stars and such objects will be missed in color-selected samples for blue stars. Only a reddening-corrected sample, which requires distance estimates, will allow candidates to be selected in regions with significant extinction. However, particularly in dense Galactic plane regions, Gaia DR2 data often provide bad astrometry, resulting in many spurious sources. Therefore, hot subdwarfs selected based on absolute magnitudes with reddening corrections are currently done best outside the Galactic plane with no crowding (Geier et al. 2019), but misses these hot subdwarfs at low Galactic latitudes that have evolved from a young stellar population and progenitor masses $>2 M_{\odot}$.

Further discoveries and extended studies using reddeningcorrected samples are needed for space density calculations and to understand whether Roche lobe-filling hot subdwarfs are more common among shell-burning hot subdwarfs rather than He core-burning hot subdwarf stars.

\section{Conclusions and Summary}

ZTF J2055+4651 was discovered as a $P_{\text {orb }}=56.34785(26)$ minute variable with a light-curve shape similar to ZTF J2130 +4420 . Follow-up observations show that ZTF J2055+4651 is the second Roche lobe-filling hot subdwarf binary known today. High signal-to-noise ratio photometry obtained with HiPERCAM in combination with Gemini spectroscopy constrain the system parameters to a mass ratio $q=M_{\mathrm{sdOB}} / M_{\mathrm{WD}}=0.60 \pm 0.03$, a mass for the $\mathrm{sdOB} M_{\mathrm{sdOB}}=0.41 \pm 0.04 M_{\odot}$, and a WD companion mass $M_{\mathrm{WD}}=0.68 \pm 0.05 M_{\odot}$. Additionally, the HiPERCAM light curve revealed a weak $(\approx 1 \%)$ eclipse of the hot WD that allowed us to constrain its blackbody temperature to $63,000 \pm 10,000 \mathrm{~K}$.

We use the stellar evolution code MESA to compute the evolution of the system and find that the hot subdwarf formed 
in a common envelope phase at a period of $\approx 140$ minutes and filled its Roche lobe close to the observed period when the hot subdwarf expanded during residual hydrogen shell burning. We predict that the binary will transfer mass for $\approx 1$ Myr and we currently observe the binary in its first $10 \%$ of this phase. Our models predict a mass transfer rate of more than $10^{-9} M_{\odot} \mathrm{yr}^{-1}$. Due to its high total mass the system will merge in $\approx 30 \mathrm{Myr}$ and either explode as subluminous type Ia supernova (Perets et al. 2019; Zenati et al. 2019) or merge and form a massive single WD (Webbink 1984; Saio 2008; Clayton 2012; Schwab 2019).

ZTF J2055+4651 and ZTF J2130+4420 are the only known binaries with a Roche lobe-filling hot subdwarf donor. Both systems have similar properties and we see mass transfer during the short-lived hydrogen shell-burning phase compared to the $\mathrm{He}$ core-burning phase, which lasts significantly longer. The most likely explanation is a selection criteria bias against more typical hot subdwarfs with $T_{\text {eff }} \approx 25,000 \mathrm{~K}$ due to severe reddening at low Galactic latitudes. Future studies require reddening-corrected samples. We predict that a substantial population of He coreburning Roche lobe-filling hot subdwarf binaries can still be discovered at low Galactic latitudes in young stellar populations.

Based on observations obtained with the Samuel Oschin Telescope 48-inch at the Palomar Observatory as part of the Zwicky Transient Facility project. ZTF is supported by the National Science Foundation under grant No. AST-1440341 and a collaboration including Caltech, IPAC, the Weizmann Institute for Science, the Oskar Klein Center at Stockholm University, the University of Maryland, the University of Washington, Deutsches Elektronen-Synchrotron and Humboldt University, Los Alamos National Laboratories, the TANGO Consortium of Taiwan, the University of Wisconsin at Milwaukee, and Lawrence Berkeley National Laboratories. Operations are conducted by COO, IPAC, and UW.

Based on observations obtained at the international Gemini Observatory, proposal ID GN-2019B-FT-102, a program of NSF's OIR Lab, which is managed by the Association of Universities for Research in Astronomy (AURA) under a cooperative agreement with the National Science Foundation. on behalf of the Gemini Observatory partnership: the National Science Foundation (United States), National Research Council (Canada), Agencia Nacional de Investigación y Desarrollo (Chile), Ministerio de Ciencia, Tecnología e Innovación (Argentina), Ministério da Ciência, Tecnologia, Inovações e Comunicações (Brazil), and Korea Astronomy and Space Science Institute (Republic of Korea). The authors thank the staff at the Gemini-North observatory for performing the observations in service mode.

Some results presented in this Letter are based on observations made with the Shane telescope. Research at Lick Observatory is partially supported by a generous gift from Google.

Based on observations made with the Gran Telescopio Canarias (GTC), installed at the Spanish Observatorio del Roque de los Muchachos of the Instituto de Astrofísica de Canarias, in the island of La Palma.

This research was supported in part by the National Science Foundation through grant ACI-1663688, and at the KITP by grant PHY-1748958. This research benefited from interactions that were funded by the Gordon and Betty Moore Foundation through grant GBMF5076.
We acknowledge the use of the Center for Scientific Computing supported by the California NanoSystems Institute and the Materials Research Science and Engineering Center (MRSEC) at UC Santa Barbara through NSF DMR 1720256 and NSF CNS 1725797.

HiPERCAM and VSD are funded by the European Research Council under the European Union's Seventh Framework Programme (FP/2007-2013) under ERC-2013-ADG grant agreement No. 340040 (HiPERCAM).

T.R.M. was supported by a grant from the United Kingdom's Science and Technology Facilities Council. P.S. acknowledges support from NSF grant AST-1514737. E.S.P.'s research was funded in part by the Gordon and Betty Moore Foundation through grant GBMF5076. D.S. acknowledges support by the Deutsche Forschungsgemeinschaft through grant HE1356/70-1.

This work has made use of data from the European Space Agency (ESA) mission Gaia (https://www.cosmos.esa.int/ gaia), processed by the Gaia Data Processing and Analysis Consortium (DPAC, https://www.cosmos.esa.int/web/gaia/ dpac/consortium). Funding for the DPAC has been provided by national institutions, in particular the institutions participating in the Gaia Multilateral Agreement.

This work benefited from a workshop held at DARK in 2019 July that was funded by the Danish National Research Foundation (DNRF132). We thank Josiah Schwab for his efforts in organizing this.

Facilities: PO:1.2 m (ZTF), Gemini:Gillett (GMOS), Shane (KAST), GTC (HiPERCAM).

Software: Lpipe (Perley 2019), Gatspy (VanderPlas \& Ivezić 2015; Vanderplas 2015), FITSB2 (Napiwotzki et al. 2004), LCURVE (Copperwheat et al. 2010), emcee (ForemanMackey et al. 2013), MESA (Paxton et al. 2011, 2013, 2015, 2018, 2019), Matplotlib (Hunter 2007), Astropy (Astropy Collaboration et al. 2013, 2018), Numpy (Oliphant 2015), ISIS (Houck \& Denicola 2000), TLUSTY (Hubeny \& Lanz 1995).

\section{ORCID iDs}

Thomas Kupfer (iD https://orcid.org/0000-0002-6540-1484 Evan B. Bauer (1) https://orcid.org/0000-0002-4791-6724 Kevin B. Burdge (ib https://orcid.org/0000-0002-7226-836X Jan van Roestel (i) https://orcid.org/0000-0002-2626-2872 Eric C. Bellm (1D https://orcid.org/0000-0001-8018-5348 Jim Fuller (iD https://orcid.org/0000-0002-4544-0750 JJ Hermes (i) https://orcid.org/0000-0001-5941-2286 Thomas R. Marsh (i) https://orcid.org/0000-0002-2498-7589 Shrinivas R. Kulkarni (i) https://orcid.org/0000-00015390-8563

E. S. Phinney (1D https://orcid.org/0000-0002-9656-4032 Thomas A. Prince (iD https://orcid.org/0000-0002-8850-3627 Paula Szkody (iD https://orcid.org/0000-0003-4373-7777 Yuhan Yao (iD https://orcid.org/0000-0001-6747-8509 Andreas Irrgang (i) https://orcid.org/0000-0002-0465-3725 Ulrich Heber (iD https://orcid.org/0000-0001-7798-6769 Vik S. Dhillon (iD https://orcid.org/0000-0003-4236-9642 Gabriel Murawski (i) https://orcid.org/0000-0001-7809-1457 Dmitry A. Duev (1) https://orcid.org/0000-0001-5060-8733 Matthew J. Graham (iD https://orcid.org/0000-0002-3168-0139

Russ R. Laher (iD https://orcid.org/0000-0003-2451-5482 S. P. Littlefair (10) https://orcid.org/0000-0001-7221-855X A. A. Mahabal (i) https://orcid.org/0000-0003-2242-0244 Frank J. Masci (iD https://orcid.org/0000-0002-8532-9395 Ben Rusholme (iD https://orcid.org/0000-0001-7648-4142 
David L. Shupe (iD https://orcid.org/0000-0003-4401-0430

Maayane T. Soumagnac (ib https://orcid.org/0000-00016753-1488

\section{References}

Allen, C., \& Santillan, A. 1991, RMxAA, 22, 255

Astropy Collaboration, Price-Whelan, A. M., Sipőcz, B. M., et al. 2018, AJ, 156,123

Astropy Collaboration, Robitaille, T. P., Tollerud, E. J., et al. 2013, A\&A, 558, A33

Bailer-Jones, C. A. L., Rybizki, J., Fouesneau, M., Mantelet, G., \& Andrae, R. 2018, AJ, 156, 58

Bellm, E. C., Kulkarni, S. R., Barlow, T., et al. 2019b, PASP, 131, 068003

Bellm, E. C., Kulkarni, S. R., Graham, M. J., et al. 2019a, PASP, 131, 018002

Bloemen, S., Marsh, T. R., Østensen, R. H., et al. 2011, MNRAS, 410, 1787

Brooks, J., Bildsten, L., Marchant, P., \& Paxton, B. 2015, ApJ, 807, 74

Burdge, K. B., Coughlin, M. W., Fuller, J., et al. 2019, Natur, 571, 528

Chambers, K. C., Magnier, E. A., Metcalfe, N., et al. 2016, arXiv:1612.05560

Claret, A., \& Bloemen, S. 2011, A\&A, 529, A75

Claret, A., Cukanovaite, E., Burdge, K., et al. 2020, A\&A, 634, A93

Clayton, G. C. 2012, JAVSO, 40, 539

Copperwheat, C. M., Marsh, T. R., Dhillon, V. S., et al. 2010, MNRAS, 402, 1824

Coughlin, M. W., Burdge, K., Phinney, E. S., et al. 2020, MNRAS, 494, L91 Dhillon, V., Dixon, S., Gamble, T., et al. 2018, Proc. SPIE, 10702, 107020L

Dhillon, V. S., Marsh, T. R., Bezawada, N., et al. 2016, Proc. SPIE, 9908, 99080Y

Evans, D. W., Riello, M., De Angeli, F., et al. 2018, A\&A, 616, A4

Fitzpatrick, E. L., Massa, D., Gordon, K. D., Bohlin, R., \& Clayton, G. C. 2019, ApJ, 886, 108

Foreman-Mackey, D., Hogg, D. W., Lang, D., \& Goodman, J. 2013, PASP, 125,306

Gaia Collaboration, Brown, A. G. A., Vallenari, A., et al. 2018, A\&A, 616, A1

Gaia Collaboration, Prusti, T., de Bruijne, J. H. J., et al. 2016, A\&A, 595, A1

Geier, S., Hirsch, H., Tillich, A., et al. 2011, A\&A, 530, A28

Geier, S., Marsh, T. R., Wang, B., et al. 2013, A\&A, 554, A54

Geier, S., Raddi, R., Gentile Fusillo, N. P., \& Marsh, T. R. 2019, A\&A, 621, A38

Gontcharov, G. A. 2016, Ap, 59, 548

Graham, M. J., Drake, A. J., Djorgovski, S. G., Mahabal, A. A., \& Donalek, C. 2013, MNRAS, 434, 2629

Graham, M. J., Kulkarni, S. R., Bellm, E. C., et al. 2019, PASP, 131, 078001

Green, G. M., Schlafly, E. F., Zucker, C., Speagle, J. S., \& Finkbeiner, D. P. 2019, arXiv:1905.02734

Han, Z., Podsiadlowski, P., Maxted, P. F. L., \& Marsh, T. R. 2003, MNRAS, 341, 669

Heber, U. 1986, A\&A, 155, 33

Heber, U. 2009, ARA\&A, 47, 211

Heber, U. 2016, PASP, 128, 082001

Heber, U., Irrgang, A., \& Schaffenroth, J. 2018, OAst, 27, 35

Houck, J. C., \& Denicola, L. A. 2000, in ASP Conf. Ser. 216, Astronomical Data Analysis Software and Systems IX, ed. N. Manset, C. Veillet, \& D. Crabtree (San Francisco, CA: ASP), 591

Hubeny, I., \& Lanz, T. 1995, ApJ, 439, 875
Hunter, J. D. 2007, CSE, 9, 90

Iben, I., Jr., \& Tutukov, A. V. 1991, ApJ, 370, 615

Irrgang, A., Kreuzer, S., Heber, U., \& Brown, W. 2018, A\&A, 615, L5

Irrgang, A., Wilcox, B., Tucker, E., \& Schiefelbein, L. 2013, A\&A, 549, A137

Kupfer, T., Bauer, E. B., Marsh, T. R., et al. 2020, ApJ, 891, 45

Kupfer, T., Ramsay, G., van Roestel, J., et al. 2017a, ApJ, 851, 28

Kupfer, T., van Roestel, J., Brooks, J., et al. 2017b, ApJ, 835, 131

Kurucz, R. L. 1996, in ASP Conf. Ser. 108, M.A.S.S., Model Atmospheres and

Spectrum Synthesis, ed. S. J. Adelman, F. Kupka, \& W. W. Weiss (San Francisco, CA: ASP), 160

Loeb, A., \& Gaudi, B. S. 2003, ApJL, 588, L117

Lomb, N. R. 1976, Ap\&SS, 39, 447

Maíz Apellániz, J., \& Weiler, M. 2018, A\&A, 619, A180

Masci, F. J., Laher, R. R., Rusholme, B., et al. 2019, PASP, 131, 018003

Maxted, P. f. L., Heber, U., Marsh, T. R., \& North, R. C. 2001, MNRAS, 326, 1391

Napiwotzki, R., Karl, C. A., Lisker, T., et al. 2004, Ap\&SS, 291, 321

Naslim, N., Jeffery, C. S., Hibbert, A., \& Behara, N. T. 2013, MNRAS, 434,1920

Nomoto, K. 1982, ApJ, 253, 798

Nomoto, K., Saio, H., Kato, M., \& Hachisu, I. 2007, ApJ, 663, 1269

Odenkirchen, M., \& Brosche, P. 1992, AN, 313, 69

Oliphant, T. E. 2015, Guide to NumPy (2nd ed.; USA: CreateSpace)

Pauli, E.-M., Napiwotzki, R., Heber, U., Altmann, M., \& Odenkirchen, M. 2006, A\&A, 447, 173

Paxton, B., Bildsten, L., Dotter, A., et al. 2011, ApJS, 192, 3

Paxton, B., Cantiello, M., Arras, P., et al. 2013, ApJS, 208, 4

Paxton, B., Marchant, P., Schwab, J., et al. 2015, ApJS, 220, 15

Paxton, B., Schwab, J., Bauer, E. B., et al. 2018, ApJS, 234, 34

Paxton, B., Smolec, R., Schwab, J., et al. 2019, ApJS, 243, 10

Perets, H. B., Zenati, Y., Toonen, S., \& Bobrick, A. 2019, arXiv:1910.07532

Perley, D. A. 2019, PASP, 131, 084503

Piersanti, L., Tornambé, A., \& Yungelson, L. R. 2014, MNRAS, 445, 3239

Romero, A. D., Kepler, S. O., Joyce, S. R. G., Lauffer, G. R., \& Córsico, A. H. 2019, MNRAS, 484, 2711

Saio, H. 2008, in ASP Conf. Ser. 391, Hydrogen-Deficient Stars, ed. A. Werner \& T. Rauch (San Francisco, CA: ASP), 69

Sandoval, L. R., Murawski, G., Copperwheat, C., \& Maccarone, T. 2020, ATel, 13444, 1

Savonije, G. J., de Kool, M., \& van den Heuvel, E. P. J. 1986, A\&A, 155, 51 Scargle, J. D. 1982, ApJ, 263, 835

Schwab, J. 2019, ApJ, 885, 27

Stroeer, A., Heber, U., Lisker, T., et al. 2007, A\&A, 462, 269

Townsley, D. M., \& Bildsten, L. 2003, ApJL, 596, L227

Tutukov, A. V., \& Fedorova, A. V. 1989, SvA, 33, 606

Tutukov, A. V., \& Yungelson, L. R. 1990, SvA, 34, 57

Vanderplas, J. 2015, gatspy: General tools for Astronomical Time Series in Python, v0.3.0, Zenodo, doi:10.5281/zenodo.14833

VanderPlas, J. T., \& Ivezić, v. 2015, ApJ, 812, 18

Vennes, S., Kawka, A., O’Toole, S. J., Németh, P., \& Burton, D. 2012, ApJL, 759, L25

Webbink, R. F. 1984, ApJ, 277, 355

Wheatley, P. J., Mauche, C. W., \& Mattei, J. A. 2003, MNRAS, 345, 49

Wolf, W. M., Bildsten, L., Brooks, J., \& Paxton, B. 2013, ApJ, 777, 136

Yungelson, L. R. 2008, AstL, 34, 620

Zenati, Y., Toonen, S., \& Perets, H. B. 2019, MNRAS, 482, 1135 